\title{
PERFIL EPIDEMIOLÓGICO DAS VÍTIMAS DE TRAUMATISMO CRANIENCEFÁLICO ATENDIDAS PELO SAMU-SALVADOR
}

\author{
EPIDEMIOLOGICAL PROFILE OF TRAUMATIC BRAIN \\ INJURY VICTIMS ASSISTED BY SAMU-SALVADOR
}

\author{
Helena Fraga-Maia*, Marcio Roberto Coelho Santos**
}

Autora para correspondência: Helena Fraga-Maia - helenafragamaia@gmail.com

*Professora adjunta na Universidade do Estado da Bahia

**Pós Graduado em Urgência e Emergência Pré-Hospitalar e Intra-Hospitalar. Enfermeiro no SAMU-Salvador.

\section{R E S U M O}

\begin{abstract}
Introdução: No Brasil, o trauma aparece como grave problema de saúde, sendo o traumatismo craniencefálico (TCE), o de maior índice de morbimortalidade. Estima-se que o tempo entre a ocorrência do trauma, o diagnóstico e o tratamento adequado seja primordial para a recuperação do paciente, principalmente na primeira hora, chamada de Golden hour. Em Salvador, o SAMU é o serviço responsável por esse atendimento e o monitoramento da frequência de agravos e de fatores associados aos traumas pode contribuir para a efetivação de melhoras no atendimento. Objetivo: caracterizar - perfil epidemiológico das ocorrências das vítimas de TCE atendidas pelo SAMU-Salvador e alguns aspectos relativos ao serviço prestado, no período de outubro de 2006 a dezembro de 2010 . Métodos: Realizou-se um estudo descritivo, avaliando-se dados secundários de todos os registros de ocorrência de vítimas de TCE atendidas pelo SAMU, em Salvador-Bahia-Brasil. Resultados: Foram analisados 105 registros de vítimas que na sua maioria eram do sexo masculino $(74,3 \%)$ e tinham entre 21 a 40 anos $(32,1 \%)$. Queda foi a principal etiologia dos TCE $(36,2 \%)$, seguida do atropelamento (19,0\%). Os dias de quinta-feira a domingo apresentaram maior frequência de vítimas $(71,4 \%)$. Observou-se um aumento dos coeficientes de prevalência das causas externas e do TCE ao longo dos anos. Conclusão: O perfil epidemiológico das vítimas de TCE atendidas pelo SAMU-Salvador foi formado principalmente por indivíduos jovens, do sexo masculino e a população economicamente ativa. Os resultados apontam para a necessidade de melhorar o sistema de notificação do SAMU
\end{abstract}

Palavras-chave: Traumatismo Craniencefálico; Acidentes; Violência; Serviço de Atendimento Móvel de Urgência - SAMU. 
Introduction: In Brazil, trauma appears as a serious health problem, where traumatic brain injury (TBI) has the highest morbidity and mortality rates. It is estimated that the time between the occurrence of trauma and proper diagnosis and treatment is essential for patient recovery, particularly during the first hour, called Golden hour. In Salvador, the SAMU is the service responsible for this assistance and the monitoring of frequencies of disorders and factors associated to trauma could contribute towards attaining improvements in this assistance. Objective: characterize the epidemiological profile of the occurrences of TBI victims assisted by the SAMU-Salvador and some aspects related to the service rendered, during the period of October 2006 to December 2010. Method: A descriptive study, evaluating secondary data of all the incidence reports of TBI victims assisted by SAMU, in Salvador-Bahia-Brazil. Results: There were 105 registers of victims analyzed, where most were of the male gender $(74.3 \%)$ of ages between 21 and 40 year $(32.1 \%)$. The main cause of the TBI was from falls $(36.2 \%)$, followed by being run over (19.0\%). Thursdays and Sundays appeared with greater frequency (71.4\%). An increase was observed in the coefficients of the prevalence for external causes and TBI throughout the years. Conclusion: The epidemiological profile of the victims of TBI served by the SAMU-Salvador was formed mainly young economically active male individuals. The results point out to the need for improvement in the notification system o the SAMU.

Key words: Traumatic brain injury; Accidents. Violence; Mobile Emergency Care Service (SAMU). 


\section{INTRODUÇÃO}

Traumatismo craniencefálico (TCE) pode ser definido como um insulto no cérebro, de natureza não degenerativa ou congênita, mas causada por uma força externa física que pode produzir uma diminuição ou alteração do nível de consciência e resultar em uma deficiência nas habilidades cognitivas ou no funcionamento físico'. Pode também resultar em distúrbios emocionais e comportamentais e todas as alterações podem ser tanto temporárias quanto permanentes. É considerada como a causa mais importante de incapacidade entre jovens e a mais frequente causa neurológica de morbidade e mortalidade ${ }^{2}$. Mesmo para os que sobrevivem às lesões iniciais e são atendidos em departamentos de emergência, a mortalidade atribuível ao TCE é de, aproximadamente, $35 \%{ }^{3}$.

Diante deste panorama e com o objetivo de prestar assistência em um tempo resposta menor, de melhorar o prognóstico do paciente e de ajudar no atendimento hospitalar, vem sendo, cada vez mais frequente, a existência dos serviços de Atendimento Pré-Hospitalar (APH) nas várias cidades do Brasil. Em 2003 foram publicadas duas portarias que normatizam os serviços de APH: a 1863 GM, que institui a Política Nacional de Atenção às Urgências, sendo um de seus componentes o atendimento préhospitalar móvel, e a 1864 GM, que oficializa a implantação do Serviço de Atendimento Móvel de Urgência (SAMU-192) em municípios e regiões de todo o território brasileiro ${ }^{4,5}$.

O APH caracteriza-se por prestar assistência às pessoas em situações de agravos no local onde tais eventos ocorrem, logo, possibilita $\circ$ atendimento precoce e adequado, favorecendo o prognóstico da vítima. Esse modelo de assistência pode influenciar positivamente na redução das taxas de morbimortalidade por trauma e tem sido validado por gestores do Sistema Único de Saúde (SUS) das esferas federal, estadual e municipal com reflexos na ampliação de recursos físicos e humanos ${ }^{6}$. Em 2010, o SAMU já funcionava em 1468 municípios brasileiros distribuídos por todos os Estados, com 157 centrais de regulação médica, atuando com equipes especializadas ${ }^{7}$.

Recomenda-se que a produção de dados de urgência e emergência seja utilizada para elaboração de informações epidemiológicas de base descritiva dos serviços de saúde. A existência de dados epidemiológicos sobre as vítimas de TCE e o serviço prestado a essas vítimas pelo $\mathrm{APH}$ é essencial para $\circ$ serviço de saúde, tanto para $\circ$ nível de planejamento, quanto para o de execução do serviço, constituindo-se em um importante instrumento norteador das políticas de organização e estruturação do SAMU, entretanto, não há um sistema de informação oficial para armazenamento dos dados das ocorrências atendidas ${ }^{4}$.

Por se tratar de uma estratégia recente e pela ausência de instrumento de coleta de dados em âmbito nacional, existe uma escassez de estudos que descrevam a demanda e $\circ$ atendimento do SAMU. Considerando-se que o monitoramento da frequência de agravos e de fatores associados aos traumas pode contribuir para a efetivação de melhorias no atendimento à população, realizou-se o presente estudo com o objetivo de caracterizar - perfil epidemiológico das ocorrências das vítimas de TCE atendidas pelo SAMU-Salvador e alguns aspectos relativos ao serviço prestado, no período de outubro de 2006 a dezembro de 2010.

\section{MÉTODOS}

Realizou-se um estudo descritivo, utilizando-se dados secundários extraídos da base de dados do SAMU-Salvador, avaliando-se todos os registros de ocorrência de vítimas de TCE assistidas pelo SAMU, a partir de outubro de 2006 a dezembro de 2010 em Salvador, Bahia, Brasil. Para selecionar as ocorrências com diagnóstico de TCE, dentro do universo dos registros das ocorrências de traumas e de causas externas, inicialmente, utilizou-se como filtro, os descritores "cabeça" OR "encéfalo" $O R$ "face" OR "nuca" OR "crânio", digitados no campo de decisão técnica dos registros da central de regulação do SAMU-Salvador.

O ano de início do período do estudo coincidiu com 
- ano de existência de dados no novo programa da base de dados do SAMU-Salvador. Os dados foram extraídos de primeiro de outubro de 2006 a 31 de dezembro de 2010. Para fins de análise, o tempo de estudo de quatro anos e três meses foi dividido em dois períodos, o primeiro período que vai de primeiro de outubro de 2006 a 31 de dezembro de 2008 e o segundo período de primeiro de janeiro de 2009 a 31 de dezembro de 2010. Todos os campos sem preenchimento foram classificados como sem informação e todos os registros que não apresentavam o campo de diagnóstico e da data do atendimento preenchidos foram excluídos.

Para a análise do perfil epidemiológico das ocorrências de TCE, calculou-se o coeficiente de prevalência de causas externas e de traumatismo craniencefálico, utilizando-se a população estimada pelo IBGE, segundo ano e sexo ${ }^{8}$. Para a faixa etária foram utilizados intervalos a cada 10 anos. Etiologia foi categorizada em agressão, atropelamento, automobilístico, moto, Projétil por Arma de Fogo (PAF) e queda. O período do dia foi definido em manhã, tarde, noite e madrugada. Os dias da semana foram colapsados de segunda a quartafeira e de quinta-feira a domingo. Os trimestres foram computados de novembro a janeiro, fevereiro a abril, maio a julho e agosto a outubro. O local da ocorrência foi definido pelos Distritos Sanitário, a saber Cento Histórico, Itapagipe, São Caetano/ Valéria, Liberdade, Brotas, Barra/Rio Vermelho, Boca do Rio, Itapuã, Cabula/Beiru, Pau da Lima, Subúrbio Ferroviário e Cajazeiras. As unidades de atendimento foram definidas em básicas ou avançadas.

O programa estatístico utilizado para a construção do banco de dados foi o Epi-info (v. 6.0) e para a análise dos dados foi empregado o STATA (v.12.0) onde foram calculadas frequências absolutas e relativas para descrição das variáveis de interesse. Este estudo foi aprovado pelo Comitê de Ética em Pesquisa da Secretaria de Saúde do Estado da Bahia (Registro 006/2011/CEP-SESAB, aprovado em 08 de abril de 2011).

foram atendidas por unidades avançadas. Vale salientar que no campo de registro sobre o tipo de unidade que prestou $\circ$ atendimento, para 21 $(20,0 \%)$ indivíduos, não havia informação (Tabela 1). O tempo para chegada no local de atendimento à vítima variou de 1 a 185 minutos com média de $20,6 \pm 22,4$ e mediana de 15 minutos. Enquanto o tempo de atendimento realizado do local de ocorrência até a chegada no local de referência variou de 1 a 1038 minutos com tempo médio de $148,9 \pm 133,6$ e mediana de 117 minutos (dados não tabulados). atendimento, detectou-se que 66 (62,9\%) vítimas foram atendidas por unidade básica e $18(17,1 \%)$

Tabela 1. Características das vítimas de traumatismo craniencefálico e da assistência prestada pelo Serviço de Atendimento Móvel de Urgência - SAMU/SALVADOR, de outubro/2006 a dezembro/2010, Salvador, Bahia.

\begin{tabular}{lcr}
\hline Variáveis & $\mathbf{n = 1 0 5}$ & $\%$ \\
\hline Sexo & & \\
Feminino & 23 & 21,9 \\
Masculino & 78 & 74,3 \\
Sem informação & 4 & 3,8 \\
\hline
\end{tabular}


Tabela 1. Características das vítimas de traumatismo craniencefálico e da assistência prestada pelo Serviço de Atendimento Móvel de Urgência - SAMU/SALVADOR, de outubro/2006 a dezembro/2010, Salvador, Bahia.

(continuação)

\begin{tabular}{lcc}
\hline Variáveis & $\mathrm{n}=105$ & $\%$ \\
\hline Faixa etária & 1 & 1,0 \\
$0-10$ anos & 9 & 8,6 \\
$11-20$ anos & 18 & 17,1 \\
$21-30$ anos & 15 & 14,3 \\
$31-40$ anos & 8 & 7,6 \\
$41-50$ anos & 7 & 6,7 \\
$51-60$ anos & 12 & 11,4 \\
61 anos ou mais & 35 & 33,3 \\
Sem informação & & \\
Etiologia & 17 & 16,2 \\
Agressão & 20 & 19,0 \\
Atropelamento & 8 & 7,6 \\
Automobilístico & 12 & 11,4 \\
Moto & 10 & 9,5 \\
PAF & 38 & 36,2 \\
Queda & & 62,9 \\
& 66 & 17,1 \\
Unidade de Atendimento & 18 & 20,0 \\
Unidade Básica & 21 & \\
Unidade Avançada & & \\
Sem informação & &
\end{tabular}

Com relação ao período do dia em que houve a solicitação, observou-se que o noturno foi $\circ$ mais frequente com $37(35,2 \%)$ casos, seguido do vespertino $(27,6 \%)$, e da madrugada com 21 $(20,0 \%)$ ocorrências. Quanto ao dia da semana verificou-se que de quinta-feira a domingo foram os dias em que houve mais solicitações $(71,4 \%)$. Quanto ao período do ano, os meses de novembro a janeiro, seguido pelos de fevereiro a abril foram os que acumularam mais vítimas $32,4 \%$ e $28,6 \%$ respectivamente. Quanto à distribuição geográfica, utilizou-se os distritos sanitários (DS) como unidade de medida. O DS Cabula/Beirú foi o que apresentou maior frequência com 17 (16,2\%) vítimas, seguido de Itapuã e São Caetano/Valéria com 13 (12,4\%) ocorrências cada e Itapagipe com $12(11,4 \%)$ eventos (Tabela 2).

Tabela 2. Características da assistência prestada pelo Serviço de Atendimento Móvel de Urgência - SAMU/SALVADOR, de outubro/2006 a dezembro/2010, Salvador, Bahia.

\begin{tabular}{lcc}
\hline Varióveis & $\mathrm{n}=105$ & $\%$ \\
\hline Período do Dia & 18 & \\
Manhã & 29 & 27,1 \\
Tarde & 37 & 35,2 \\
Noite & 21 & 20,0 \\
Madrugada & & \\
Dia da semana & 30 & 28,6 \\
Segunda-feira - Quarta-feira & 75 & 71,4 \\
Quinta-feira - Domingo & & \\
\hline
\end{tabular}


Tabela 2. Características da assistência prestada pelo Serviço de Atendimento Móvel de Urgência - SAMU/SALVADOR, de outubro/2006 a dezembro/2010, Salvador, Bahia.

(continuação)

\begin{tabular}{lcr}
\hline Variáveis & $\mathrm{n}=105$ & $\%$ \\
\hline Trimestre & 34 & 32,4 \\
Novembro - Janeiro & 30 & 28,6 \\
Fevereiro - Abril & 22 & 21,0 \\
Maio - Julho & 19 & 18,1 \\
Agosto - Outubro & & \\
& & \\
Distrito sanitário & 9 & 8,6 \\
Centro Histórico & 12 & 11,4 \\
Itapagipe & 13 & 12,4 \\
São Caetano/Valéria & 2 & 1,9 \\
Liberdade & 8 & 7,6 \\
Brotas & 11 & 10,5 \\
Barra/Rio Vermelho & 2 & 1,9 \\
Boca do Rio & 13 & 12,4 \\
Itapuã & 17 & 16,2 \\
Cabula/Beirú & 4 & 3,8 \\
Pau da Lima & 11 & 10,5 \\
Subúrbio Ferroviário & 3 & 2,9 \\
Cajazeiras & & \\
\hline
\end{tabular}

Na Tabela 3 apresentam-se os coeficientes de prevalência de causas externas e de TCE para vitimas assistidas entre 2006 a 2010 e a distribuição dos TCE de acordo com $\circ$ sexo. Observa-se a evolução dos indicadores de 164,8 em 2006 para $1.168,4$ em 2010 e a prevalência mais elevada para homens quando comparado com as mulheres.

Tabela 3. Coeficiente de prevalência das vítimas de causas externas e traumatismo craniencefálico, assistidas pelo Serviço de Atendimento Móvel de Urgência-SAMU/SALVADOR, segundo sexo, de outubro/2006 a dezembro/2010, Salvador, Bahia.

\begin{tabular}{|c|c|c|c|c|c|}
\hline \multirow[t]{2}{*}{ Coeficientes de Prevalência* } & \multicolumn{5}{|c|}{ Ano } \\
\hline & 2006 & 2007 & 2008 & 2009 & 2010 \\
\hline Causa Externas & 164,8 & 835,7 & 850,1 & 950,5 & 1168,4 \\
\hline TCE & 0,2 & 1,0 & 0,5 & 0,9 & 1,1 \\
\hline TCE em mulheres & 0,1 & 0,3 & 0,3 & 0,4 & 0,4 \\
\hline TCE em homens & 0,3 & 1,8 & 0,7 & 1,4 & 1,8 \\
\hline
\end{tabular}

Na Tabela 4 encontram-se dispostas as informações acerca da etiologia dos TCE de acordo com o sexo, faixa etária, e unidade de atendimento, de outubro/2006 a dezembro/2010. Observa-se, para cada etiologia, frequencia mais elevada de eventos para os homens, exceto para acidentes automobilísticos com discreta elevação para as mulheres. Os picos de frequencia foram encontrados para as faixas etárias mais velhas, de 51 a 60 $(71,4 \%)$ e 61 anos em mais $(83,3 \%)$, seguidos pelas faixas de 21 a 30 anos $(44,4 \%)$ e 31 a $40(46,7 \%)$. Com relação ao tipo de unidade de atendimento verificou-se que as vítimas atendidas por unidades básicas foram aquelas que sofreram agressão (100\%), 93,8\% por atropelamento, $82,1 \%$ por queda e $77,8 \%$ por acidente motociclístico. Os atendimentos em unidades avançadas foram mais frequentes para as vitimas de $\operatorname{PAF}(40,0 \%)$, acidente automobilístico $(75,0 \%)$ e quedas $(13,2 \%)$. 
Tabela 4. Etiologia dos traumatismos craniencefálicos assistidos pelo Serviço de Atendimento Móvel de Urgência- SAMU/ SALVADOR, segundo sexo, faixa etária e unidade de atendimento, de outubro/2006 a dezembro/2010, Salvador, Bahia.

\begin{tabular}{|c|c|c|c|c|c|c|c|c|c|c|c|c|c|}
\hline \multirow[t]{3}{*}{ Variáveis } & \multicolumn{12}{|c|}{ Etiologia } & \multirow[b]{3}{*}{$\%$} \\
\hline & \multicolumn{2}{|c|}{ Agressão } & \multicolumn{2}{|c|}{ Atropelamento } & \multicolumn{2}{|c|}{ Aułomobilístico } & \multicolumn{2}{|c|}{ Moto } & \multirow[t]{2}{*}{ PAF } & \multicolumn{3}{|c|}{ Queda } & \\
\hline & $n=15$ & $\%$ & $\mathbf{n}=\mathbf{2 0}$ & $\%$ & $n=8$ & $\%$ & $n=12$ & $\%$ & & $n=10$ & $\%$ & $n=38$ & \\
\hline \multicolumn{14}{|l|}{ Sexo } \\
\hline Feminino & 5 & 21,7 & 2 & 8,7 & 2 & 8,7 & - & - & & - & - & 14 & 60,9 \\
\hline Masculino & 10 & 12,8 & 16 & 20,5 & 6 & 7,7 & 12 & 15,4 & & 10 & 12,8 & 24 & 30,8 \\
\hline \multicolumn{14}{|l|}{ Faixa etária* } \\
\hline $0-10$ anos & - & - & 1 & 100,0 & - & - & - & - & & - & - & - & 0 \\
\hline $11-20$ anos & 1 & 11,1 & 2 & 22,2 & 2 & 22,2 & 2 & 22,2 & & - & - & 2 & 22,2 \\
\hline $21-30$ anos & 2 & 11,1 & 3 & 16,7 & 3 & 16,7 & 1 & 5,6 & & 1 & 5,6 & 8 & 44,4 \\
\hline $31-40$ anos & 5 & 33,3 & - & - & - & 0 & 3 & 20,0 & & - & - & 7 & 46,7 \\
\hline $41-50$ anos & 2 & 25,0 & 1 & 12,5 & 1 & 12,5 & 1 & 12,5 & & 1 & 12,5 & 2 & 25,0 \\
\hline $51-60$ anos & - & - & - & - & 1 & 14,3 & - & - & & 1 & 14,3 & 5 & 71,4 \\
\hline 61 anos ou mais & - & - & 1 & 8,3 & - & - & 1 & 8,3 & & - & - & 10 & 83,3 \\
\hline \multicolumn{14}{|c|}{ Unidade de Atendimento* } \\
\hline Unidade Básica & 15 & 22,7 & 15 & 22,7 & 1 & 1,5 & 7 & 10,6 & & 5 & 7,6 & 23 & 34,8 \\
\hline Unidade Avançada & - & - & 1 & 5,6 & 6 & 33,3 & 2 & 11,1 & & 4 & 22,2 & 5 & 27,8 \\
\hline
\end{tabular}

\section{DISCUSSÃO}

O perfil epidemiológico das vítimas de TCE atendidas pelo SAMU-Salvador foi formado principalmente por indivíduos jovens e do sexo masculino. Estes dados são condizentes com a literatura já que indivíduos do sexo masculino têm sido mais afetados por todas as causas externas de morbimortalidade. Vários fatores têm sido atribuídos, desde a maior exposição a comportamentos violentos e afinidade com os signos do machismo como armas e carros, à realização de trabalhos mais arriscados e ao consumo de álcool e drogas. Freitas et $\mathrm{al}^{9}$, estimaram taxa de mortalidade até seis vezes maior para o sexo masculino quando comparados com o sexo feminino. Para Gawrysznewski et $\mathrm{al}^{10}$, o risco de um homem se tornar vítima fatal de acidentes automobilísticos é 4,3 vezes maior que o da mulher.

Vários estudos corroboram os achados de que os homens são mais afetados tanto por acidentes quanto por violências e, entre eles, os mais jovens. Minayo \& Souza ${ }^{11}$, apontaram como fator predisponente para as causas externas o comportamento de risco adotado por muitos deles e, especialmente, nos grandes centros urbanos. De acordo com Szwarcwald \& Castilho' ${ }^{12}$, a adoção, pela sociedade moderna, de armas e carros como símbolo da masculinidade, expõe os indivíduos aos acidentes e homicídios. Para Souza ${ }^{13}$, os carros simbolizam poder em geral a possibilidade de transportar-se com facilidade, e ainda liberdade e velocidade. Já as armas têm $\circ$ poder de fazer com que o outro se submeta a seus desejos e interesses e também traduz a possibilidade de deixar o outro viver ou não. Esses objetos, carros e armas, participam desde muito cedo da vida dos meninos e passam a fazer parte do universo masculino.

Verifica-se, também, que as agressões por armas de fogo constituem um grande problema de saúde pública. Nos anos 90, há estimativas de que os ferimentos por arma de fogo tornaram-se a principal causa de morbimortalidade em nove capitais brasileiras, com destaque para Salvador ${ }^{14}$. Ferimentos por projéteis de arma de fogo (PAF) em crânio são traumas particularmente graves, quase sempre fatais. No presente estudo, os TCE por arma de fogo representaram $100 \%$ dos casos de agravos por violência interpessoal para homens. Adolescentes e adultos jovens, negros e de baixa renda representam o pool de vítimas, pois estes compõem o grupo mais exposto aos problemas sociais, devido à "cultura machista" ainda predominante na sociedade, muitas vezes ligados a trabalhos informais, na terceirização, desenvolvendo os serviços "mais arriscados" e exercendo atividades ilegais como o comércio e o uso de armas de fogo ${ }^{15,16}$.

As quedas, em geral, também acometem significativamente crianças de 0 a 10 anos estando 
relacionadas principalmente com deslocamentos do colo da mãe, do berço, da cama ou da escada, escada, laje, cama, muro ou árvore ${ }^{17}$. Outras vítimas frequentes deste tipo de trauma são os idosos com mais de 60 anos, em função de desvantagens impostas pela idade como distúrbios da marcha, do equilíbrio e alterações visuais ${ }^{18}$. Para esta faixa etária, as quedas são consideradas como a principal causa de TCE fatais ou não. Fatores de risco para quedas em idosos incluem também o uso de sedativos, antidepressivos, ou outras medicações psicotrópicas. Como medidas efetivas, a redução do risco de quedas pode ser empreendida com modificação ambiental e, quando possível, redução do uso destas classes medicamentosas ${ }^{19,20}$. No estudo atual, as quedas foram umas das mais importantes causas acidentais dos TCE.

Os acidentes de trânsito constituem a segunda causa mais frequente de traumatismos craniencefálicos que pode ser justificada, em parte, pelo rápido aumento do número de veículos, falta de respeito às leis de trânsito e estar sob efeito de bebidas alcoólicas. A ausência de proteção individual, como capacetes, pode também justificar as altas taxas deste tipo de morbidade para motociclistas. Para Wu et $\mathrm{al}^{21}$ que estudaram causas de TCE na China, os motociclistas e pedestres foram também os grupos mais vulneráveis. Em função do trânsito e do custo facilitado para a aquisição de motocicletas, cada vez mais adultos jovens usam este tipo de transporte nas áreas urbanas e rurais e como a fiscalização é precária em algumas áreas da cidade, muitos não usam os equipamentos de proteção obrigatórios.

Quanto aos tipos de unidade de atendimento, deve-se reiterar que 0 perfil de atendimento por parte das equipes de suporte básico cumpre com as principais finalidades do APH que é prevenir lesões secundárias, manter a estabilidade clínica das vítimas e transportar a vítima para a unidade de referência em menor tempo possível ${ }^{22}$. Destacase $\circ$ aumento de atendimento às vítimas por unidades básicas do primeiro período (19 vítimas) para o segundo (47 vítimas), sendo este aumento de $147 \%$, acompanhado também pelo aumento de atendimento das unidades avançadas. Tal fato se deve provavelmente, tanto pelo aumento da demanda, como pelo aumento da oferta, representado pelo crescimento da frota e do número de bases em posicionamento estratégicos.
Entretanto, cabe destacar aspectos relacionados à notificação.

O maior número de registros não preenchidos no campo "tipo de unidade de atendimento" no primeiro período pode estar contribuindo para esse "aumento", sendo que na realidade, isso pode não ter acontecido. Assim, mais uma vez, evidenciase a necessidade de conscientização de todos os envolvidos na notificação dos dados quanto a qualidade do registrado, de forma que estes dados representem de fato a realidade.

Com relação ao horário em que ocorreram os traumas pode-se supor que pode estar relacionado com fatores como o cansaço ao final do dia, uso de bebida alcoólica e outras drogas e também menor visibilidade no trânsito. É possível ainda, que, a existência de eventos e/ou atividades profissionais durante a noite e a infração das leis de trânsito, possam contribuir com a elevação do número de vítimas neste período. Já com relação aos dias da semana, observou-se aumento do número de vítimas de quinta a domingo, corroborando com outros estudos que demonstram esses dias da semana como os que registram maior número de vítimas por trauma ${ }^{24,25}$. Esses fatores apontados, também por Fraga-Maia ${ }^{23}$, podem ser justificados pelo maior consumo de bebidas alcoólicas, neste período da semana, tal como também relataram Pereira \& $\mathrm{Lima}^{24}$ em 2006. Com relação ao trimestre em que ocorreram os acidentes, os que apresentaram maior número de vítimas foram os que compreendem os períodos de novembro a janeiro e de fevereiro a abril. É provável que esses agravos sejam influenciados pela estação climática, no caso o verão, que traz a possibilidade de uma população flutuante de turistas por se tratar de uma capital da região nordeste do Brasil e também pelos festejos natalinos e de final do ano. Sabe-se também que $\circ$ período de férias e das festas pré e póscarnavalescas funcionam como apelo para o maior consumo de bebidas alcoólicas.

Na análise da distribuição geográfica das ocorrências dos traumas, utilizou-se os distritos sanitários como unidade de medida. $O$ distrito de Cabula/Beirú foi o de maior frequência de vítimas, seguido de ltapuã e São Caetano/Valéria. Os resultados do presente estudo corroboram os produzidos por Fraga Maia ${ }^{23}$, em 2010. Segundo esta autora, contribuíram para 
tais achados os fatores topografia do bairro, baixa escolaridade e renda, e altas taxas de desemprego entre os moradores que favorece a adoção de trabalhos terceirizados e de alto risco, e também o maior consumo de álcool e drogas.

Quanto ao tempo para chegada ao local de atendimento à vítima os resultados diferem dos apontados por outros autores, tais como Malvestio e Sousa ${ }^{25}$, que evidenciaram tempo médio de atendimento total de 37 minutos por parte das unidades e que os encontrados no presente estudo estão muito acima destes. Porém, deve-se levar em consideração que uma série de fatores influenciam nesses tempos, como por exemplo, o maior ou menor número de ocorrências no dia do atendimento, - maior ou menor número de veículos nas vias no horário da ocorrência, a gravidade clínica da vítima influenciando no tempo total de atendimento, a dificuldade de leito vago nas unidades de referência para a vítima e erros de notificação do tempo.

Esta pesquisa apresenta limitações e, dentre estas, a qualidade dos registros que não permitiu descrever as lesões e a gravidade dos traumas de crânio. Ainda assim, acredita-se que foi possível fornecer informações que podem ser úteis para o planejamento de ações preventivas e de controle para os gestores da área da saúde e para o SAMU.

\section{CONCLUSÕES}

Os resultados permitem concluir que as principais vítimas de traumatismos craniencefálicos são adultos jovens do sexo masculino e os fenômenos associados são, em geral, potencialmente evitáveis, ○ que pode contribuir para a implantação de estratégias de prevenção de acidentes. Já as informações relativas ao tempo médio para a chegada ao local do atendimento à vítima (20,6 minutos) e o tipo de unidade que mais atendeu às vítimas (básica), podem auxiliar no planejamento, na alocação de recursos e no treinamento de pessoal, ou seja, na organização da rede de urgência e emergência. Recomendam-se investimentos que viabilizem a implantação de um sistema de vigilância e monitoramento dos traumas de crânio com informações provenientes de vários setores. Tais ações podem contribuir para a redução dos fatores de risco para TCE. Para tanto, se faz necessário um maior empenho e conscientização da importância da notificação de tais dados, na construção de um sistema de informação do SAMU com dados de melhor qualidade.

\section{REFERÊNCIAS}

1. Horn LJ, Zasler ND. Medical rehabilitation of traumatic brain injury. Philadelphia: Hanley \& Belfus; 1996.

2. Brasil. Ministério da Saúde. Secretaria de Vigilância em Saúde. Impacto da violência na saúde dos brasileiros. Brasília. 2005.

3. Myburgh JA, Cooper DJ, Finfer SR, Venkatesh $B$, Jones D, Higgins A, Australasian Traumatic Brain Injury Study (ATBIS) Investigators for the Australian, New Zealand Intensive Care Society Clinical Trials Group et al. Epidemiology and 12-Month Outcomes from Traumatic Brain Injury in Australia and New Zealand. J Trauma. 2008;64(4):854862. doi: $10.1097 /$ TA.0b013e3180340e77

4. Brasil. Ministério da Saúde. GM/MS nº 1863. Institui a política nacional de atenção às urgências. Diário Oficial da União, 29 set. 2003. Brasília. 2003.

5. Brasil. Ministério da Saúde GM/MS n 1864. Institui o componente pré-hospitalar móvel da política nacional de atenção às urgências. Diário Oficial da União, 29 set. 2003. Brasília. 2003.

6. Pereira WAP, Lima MADS. O Trabalho em Equipe no Atendimento Pré-Hospitalar à Vítima de Acidente de Trânsito. Rev. Esc. Enferm. USP 2009;43(2):320-7. doi: 10.1590/S008062342009000200010

7. Brasil. Ministério da Saúde. Rede Nacional SAMU 192 [Internet]. 2011 [Acessado em 25 de maio de 2011 ]. Disponível em: http://portal. saude.gov.br/portal/saude/visualizar_texto. $\mathrm{cfm}$ ? idtxt $=36689$ \& janela $=1$ acesso 
8. Brasil. Ministério da Saúde. Informações de Saúde Demográficas e Socioeconômicas [Internet]. 2016 [Acessado em 26 de maio de 2016]. Disponível em: http://www2.datasus.gov.br/ DATASUS/index.php? area =0206\&VObj=http:// tabnet.datasus.gov.br/cgi/deftohtm.exe?ibge/cnv/ pop

9. Freitas EV. Tratado de Geriatria e Gerontologia: Rio de Janeiro: Guanabara-Koogan; 2002.

10. Gawryszewski VP, Koizumi MS, Mello Jorge MHP. As causas externas no Brasil no ano 2000: comparando a mortalidade e a morbidade. Cad Saúde Pública. 2004;20(4):995-1003. doi: $10.1590 /$ S0102-311X2004000400014

11. Minayo MCS, Souza ER. Violência sob o olhar da saúde. Rio de Janeiro: Fiocruz; 2003.

12. Szwarcwald CL, Castilho EA. Mortalidade por causas externas no estado do Rio de Janeiro no período de 1976 a 1980. Cad. Saúde Pública. 1986;2(1):19-41. doi: 10.1590/S0102$311 \times 1986000100003$

\section{Souza E. Quando viver é o grande} risco-aventura. Cad Saúde Pública. 2001;17(6):1291-1292. doi: 10.1590/S0102$311 \times 2001000600004$

14. Peres M, Santos P. Mortalidade por homicídios no Brasil na década de 90: o papel das armas de fogo. Rev. Saúde Pública. 2005;39(1)58-66. doi: $10.1590 /$ S0034-89102005000100008

\section{Ceccheto FR. Violência e estilos de} masculinidades. Rio de Janeiro: Fundação Getúlio Vargas; 2004.

16. Cecarelli P. Delinquência: resposta a um social patológico. Rev. de Psicanálise. $2001 ; 14$ (145): 5-13.

17. Melo JRT, Santana DLP, Pereira LB, Ribeiro TF. Traumatismo craniencefálico em crianças e adolescentes na cidade do Salvador - Bahia. 2006;64(4):994-996. doi: 10.1590/S0004$282 \times 2006000600020$

18. Campbell AJ, Borrie MJ, Spears GF. Risk factors for falls in a community-based prospective study of people 70 years and older. J Gerontol. 1989;44(4):M1 12-M17. doi: 10.1093/ geroni/44.4.M1 12

\section{Perracini MR, Ramos LR. Fatores associados} a quedas em uma coorte de idosos residentes na comunidade. Rev Saúde Pública. 2002;36(6):70916. doi: $10.1590 /$ S0034-89102002000700008

20. Fried LP, Tangen CM, Walston J, Newman $A B$, Hirsh $C$, Gottdiener J et al. Frailty in older adults: evidence for a phenotype. J Gerontol Biol Sci Med Sci. 2001 ;56:M1 46-57. doi: 10.1093/ gerona/56.3.M1 46

\section{Wu X, Hu J, Zhuo L, Fu C, Hui G, Wang Y et} al. Epidemiology traumatic brain injury in Eastern China 2004: a prospective large case study. J Trauma. 2008;64(5):1313-19. doi: 10.1097/ TA.0b013e318165c803

22. Pereira WAP, Lima MADS. O Trabalho em Equipe no Atendimento pré-hospitalar à vítima de acidente de trânsito. Rev. Esc. Enferm. USP 2009;43(2):320-7. doi: 10.1590/S008062342009000200010

23. Fraga-Maia H. "Vida após o trauma": do evento à funcionalidade dos sobreviventes do traumatismo craniencefálico [Doutorado em Saúde Pública]. Salvador: Universidade Federal da Bahia. Instituto de Saúde Coletiva; 2010.

24. Pereira WAP, Lima MADS. Atendimento PréHospitalar: Caracterização das Ocorrências de Acidentes de Trânsito. Acta Paul. Enf. 2006; 19(3):279-83. doi: 10.1590/S010321002006000300004

25. Malvestio MAA, Sousa RMD. Suporte avançado à vida: atendimento a vítimas de acidentes de trânsito. Rev Saúde Pública. 2002;36(5):584-9. doi: 10.1590/S0034-89102002000600007 